\title{
The AtDREB1A transcription factor up-regulates expression of a vernalization pathway gene, GmVRN1-like, delaying flowering in soybean
}

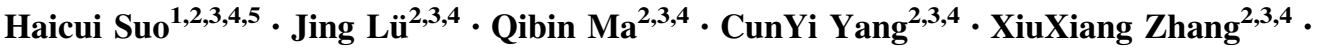 \\ Xing Meng ${ }^{2,3,4} \cdot$ Shangzhi Huang ${ }^{1} \cdot$ Hai Nian ${ }^{2,3,4}$
}

Received: 22 September 2015/Revised: 5 April 2016/Accepted: 8 April 2016/Published online: 10 May 2016

(c) The Author(s) 2016. This article is published with open access at Springerlink.com

\begin{abstract}
The dehydration-responsive element binding proteins/C-repeat binding factors (i.e., DREBs/CRT) are crucial transcription factors, they can bind the cis-elements containing the A/GCCGAC sequence in the promoter region. Overexpression of DREB genes enhances the resistance to multiple abiotic stresses, but also causes dwarfism and delayed flowering in many plant species. In this study, constitutive overexpression of AtDREB1A in
\end{abstract}

Communicated by O Ferrarese-Filho.

H. Suo and J. Lü contributed equally to this work.

Electronic supplementary material The online version of this article (doi:10.1007/s11738-016-2136-4) contains supplementary material, which is available to authorized users.

Hai Nian

hnian@scau.edu.cn

1 School of Life Sciences, Sun Yat-Sen University, No. 135, Xingang Xi Road, Guangzhou 510275, China

2 The State Key Laboratory of Agricultural and Biological Resources Protection and Utilization in the Subtropics, South China Agricultural University, Guangzhou 510642, China

3 The Key Laboratory of Plant Molecular Breeding, South China Agricultural University, Guangzhou 510642, China

4 The Guangdong Subcenter of the National Center for Soybean Improvement, College of Agriculture, South China Agricultural University, Guangzhou 510642, China

5 The Crop Research Institute, Guangdong Academy of Agriculture, Guangzhou 510642, China soybean plants caused dwarfism and delayed-flowering phenotypes. The delayed-flowering phenotype was not affected by day length and could not be reversed by exogenous gibberellic acid. The expression levels of flowering-related genes were determined by quantitative real-time RCR. The Glymallg13220 (designated as GmVRN1-like) expression was strongly induced in transgenic plants. GmVRNI-like was homologous to the Arabidopsis thaliana VRN1 gene (At3g18990). Electrophoretic mobility shift assay showed that AtDREB1A could bind the dehydration-responsive element motif, ACCGAC, in a region -157 to -186 bp upstream of GmVRNI-like. The motif shared $100 \%$ identity with the DRE sequence present in $R D 29 A$. Our results imply that the delayed-flowering of AtDREB1A-overexpressing plants might be caused by up-regulating of GmVRN1-like gene.

Keywords Soybean - AtDREB1A overexpression . GmVRN1-like · Delayed flowering

\begin{tabular}{|c|c|}
\hline \multicolumn{2}{|c|}{ Abbreviations } \\
\hline $\mathrm{ABA}$ & Abscisic acid \\
\hline AP2/EREBP & $\begin{array}{l}\text { APETALA2/ethylene responsive elemen } \\
\text { binding protein }\end{array}$ \\
\hline $\mathrm{CBF}$ & C-repeat binding factors \\
\hline $\mathrm{CO}$ & Constans \\
\hline DAE & Days after emergence \\
\hline DREB & $\begin{array}{l}\text { Dehydration-responsive element binding } \\
\text { proteins }\end{array}$ \\
\hline EMSA & Electrophoretic mobility shift assay \\
\hline FLC & Flowering locus $\mathrm{C}$ \\
\hline FT & Flowering locus $\mathrm{T}$ \\
\hline GA & Gibberellic acid \\
\hline SOC1 & Suppressor of constans 1 \\
\hline VRN1 & Vernalization 1 \\
\hline
\end{tabular}




\section{Introduction}

Abiotic stresses, including drought, salinity, and temperature have disadvantageous effects on plant development. Many of genes associated with stress resistance have been identified (Kissoudis et al. 2014). Transcription factors can regulate downstream genes by binding the cis-elements present in their promoter (Chen and Zhu 2004). The dehydration-responsive element binding proteins (DREBs) transcription factors regulate the stress-inducible genes in an abscisic acid (ABA)-independent pathway (YamaguchiShinozaki and Shinozaki 2006). The DREBs containing a conserved DNA-binding domain belong to the APETALA2/ethylene responsive element binding protein (AP2/ EREBP) superfamily. They can regulate the downstream stress-inducible genes through binding the dehydration responsive element (DRE) with core sequence A/GCCGAC present in their promoter region. This interaction regulates genes expression and enhances the tolerance to abiotic stresses (Baker et al. 1994; YamaguchiShinozaki and Shinozaki 1994; Stockinger et al. 1997).

Many studies have found that overexpression of DREB genes improve tolerance to multiple abiotic stresses in many plants such as A. thaliana, rice, wheat, maize, soybean, barley and tomato (Agarwal et al. 2006; Lata and Prasad 2011; Mizoi et al. 2012). The A. thaliana DREB genes have been well-studied. Six genes encoding CBF/ DREB1 proteins (DREBIa, DREBIb, DREB1c, DREBId, $D R E B 1$, and DREB If) have been identified (Sakuma et al. 2002; Gilmour et al. 2004). The expression of DREBs is up-regulated by low temperature and transiently contributes to enhanced tolerance (Agarwal et al. 2006). Multiple mechanisms, such as soluble sugars and proline accumulation, cold-regulated genes regulation, have been identified to be responsible for increasing freezing tolerance (Thomashow 1998, 1999; Gilmour et al. 2004).

Overexpression of DREB genes also cause dwarfism and delayed flowering (Liu et al. 1998; Gilmour et al. 2000; Hsieh et al. 2002; Dubouzet et al. 2003; Kasuga et al. 2004; Ito et al. 2006; Achard et al. 2008; Huang et al. 2009; Li et al. 2012; Suo et al. 2012). Some cases of dwarfism can be reversed by exogenous gibberellic acid (GA) treatments (Hsieh et al. 2002; Magome et al. 2004; Achard et al. 2008; Huang et al. 2009; Suo et al. 2012). Studies have also shown that dwarfism is mediated by a GA metabolic pathway (Magome et al. 2004; Achard et al. 2008; Huang et al. 2009; Suo et al. 2012). However, some instances of dwarfism cannot be reversed (Magome et al. 2004). Some delayed-flowering phenotypes can be rescued or partially rescued using exogenous GA (Achard et al. 2008; Magome et al. 2008; Huang et al. 2009). This implies that the delayed-flowering phenotype is GA-pathway dependent.
However, in some cases, delayed flowering cannot be rescued by exogenous GA treatments (Tong et al. 2009; Suo et al. 2012). Little is known at the molecular level regarding how overexpression of $D R E B$ genes causes delayed flowering. Previous studies showed that overexpression of DREB genes causes delayed flowering through activating the floral suppressor, FLOWERING LOCUS C (FLC) (Seo et al. 2009).

Flowering is the most critical event in the life cycle of angiosperm.To live and propagate, plants have evolved complicated and coordinated genetic networks responding to exogenous and endogenous signals to make sure flowering at the right time (Boss et al. 2004; Baurle and Dean 2006). In A. thaliana, there are at least four pathways regulating flowering time, including the GA, autonomous, photoperiod, and vernalization pathways (Simpson and Dean 2002; Boss et al. 2004; Bernier and Perilleux 2005; Baurle and Dean 2006). The photoperiod and vernalization pathways regulate flowering time through sensing environmental signals related to day length and low temperature, respectively. In contrast, the GA and autonomous pathways are controlled by internal signals in response to flowering (Srikanth and Schmid 2011). However, there is increasing evidence that these pathways may not act independently of each other. There exists extensive crosstalk among different pathways, ultimately affecting the downstream floral integrators including FLOWERING LOCUS T (FT), SUPPRESSOR OF CONSTANS 1 (SOC1), and $L E A F Y$. The CONSTANS (CO) and FLC regulate these integrator genes antagonistically (Mouradov et al. 2002). CONSTANS acts as a floral activator, whereas FLC acts as a floral repressor (Michaels and Amasino 1999; Lee et al. 2000; Mouradov et al. 2002).

Soybean is a major oilseed leguminous crop that has unique vegetative and floral characteristics. However, information regarding the molecular mechanisms of flower initiation and development is limited. There is increasing evidence of conservation of flowering pathways among many plant species (Hecht et al. 2005). Comparative genomic analyses demonstrated that there are conserved genes between soybean and A. thaliana (Jung et al. 2012; Kim et al. 2012). Additionally, 491 putative soybean flowering regulatory genes have been characterized (Jung et al. 2012). Previously, we observed dwarfism and delayed flowering in AtDREB1A overexpression soybean plants. The dwarfism could be rescued by GA, but delayed flowering could not (Suo et al. 2012). According to these results, we speculate that the regulatory mechanisms of dwarfism and delayed flowering are relatively independent. In this study, we investigated the response of AtDREBIA to photoperiod in transgenic and wild-type (WT) plants. We also evaluated the transcriptional levels of homologs of $A$. 
thaliana genes related to flowering using quantitative realtime polymerase chain reaction (qRT-PCR). Finally, an electrophoretic mobility shift assay (EMSA) was used to analyze the target gene of AtDREBIA. The results indicated that up-regulation of GmVRN1-like may be responsible for inducing delayed flowering in AtDREBIAoverexpressing plants.

\section{Materials and methods}

\section{Plant materials and phenotypic analysis}

Transgenic lines of soybean cultivar Huachun 5 overexpressing AtDREB1A were developed using an Agrobacterium tumefaciens-mediated method described in the previous study (Suo et al. 2012). Because the L2 plants displayed obvious phenotypic changes, the $\mathrm{T}_{3}$ plants of L2 were selected for subsequent studies. The transgenic L2 and WT soybean seeds were first germinated in sand until cotyledons emerged (about 5 days). Healthy and uniform seedlings were transferred to pots with one plant per pot and a 5-day recovery period. The seedlings were grown in growth chambers with three photoperiods, corresponding to 16,12 , and $8 \mathrm{~h}$ of light. The temperatures during growth were 28 and $24{ }^{\circ} \mathrm{C}$ during the light and dark periods. There were three replicates for each photoperiod condition. The numbers of trifoliate leaves and flowering time were recorded during the growth period.

\section{Transcriptional analyses}

To examine the transcriptional levels of soybean floweringrelated genes under different day length conditions, fully expanded leaves from transgenic L2 and WT plants were sampled at $12 \mathrm{~h}$ after dawn during the fourth trifoliate leaves stage. The detailed sampling days were listed in Online Resource 1. Total RNA of soybean was isolated using TRIzol reagent (Invitrogen, USA). $1 \mathrm{mg}$ total RNA was treated with RNase-free DNase (TaKaRa, Japan) and then was reversed using the oligo (dT) primer and M-MLV reverse transcriptase (Invitrogen, USA). The qRT-PCR was performed with a SYBR Green I kit (Bio-Rad, USA) using a CFX96 system (Bio-Rad, USA). Three biologically independent RNA samples were analyzed by qRT-PCR in triplicate. The primer efficiency have been determined with the range of $90.2-109.0 \%$ and used for calculated gene expression. The gene $\beta$-tubulin was used as internal control (Wang et al. 2012; Lü et al. 2015). The $2^{-\Delta \Delta C t}$ method was used to detect the relative expression levels of flower genes (Livak and Schmittgen 2001). According to the Bio-Rad CFX96 manufacturer's instructions, we used the calibration sample as the control, which consists of mixed- samples cDNA, the tubulin primer as well as SsoFast EvaGreen Supermix kit (Bio-Rad), the other samples were treatments. Eighty-five genes homologous to A. thaliana flowering time regulators were selected based on published information (Srikanth and Schmid 2011; Jung et al. 2012; Kim et al. 2012; Watanabe et al. 2012; Blumel et al. 2015) (Table 1). Gene information and primer sequences are provided in Online Resource 2.

\section{Electrophoretic mobility shift assay}

We generated a glutathione S-transferase (GST)AtDREB1A recombinant protein. Previous reports indicated that the DREB protein is capable of interacting with the DRE motif (A/GCCGAC) present in $r d 29 A$ (Magome et al. 2008). Therefore, the promoter of $r d 29 A$ was also prepared as an experimental control.

The length of $663 \mathrm{bp} A t D R E B 1 A$ open reading frame (ORF) was obtained from $A$. thaliana cDNA. The AtDREB IA ORF digested with BamHI/EcoRI was cloned into the pGEX-4T-2 vector. After sequencing to confirm, the vector was transformed into $E$. coli BL21. Expression of the AtDREB1A-GST fusion protein was induced using $50 \mathrm{nmol}$ isopropyl $\beta$-D-1-thiogalactopyranoside at $37{ }^{\circ} \mathrm{C}$ for $12 \mathrm{~h}$ with shaking $(150 \mathrm{rpm})$. Proteins were extracted with $4 \mathrm{ml}$ B-PER ${ }^{\mathrm{TM}}$ protein extraction reagent (Pierce, USA). We used 5'-Cy5-labeled double-stranded oligonucleotides as gel shift assay probes. The probe sequences containing the core sequence ACCGAC were as follows:

VRN1-like: 5'-ACTAGTTGTCTACCGACATGCATGT ACGTG-3'

Mutant-VRN1-like: 5'-ACTAGTT G TCTACTTATATG CATGTACGTG-3'

rd29A: 5'-GATATACTACCGACATGAGTTCCAAAA AGC-3'

Mutant-rd29A: 5'-GATATACTACTTATATGAGTTCC AAAAAGC-3' (Magome et al. 2008)

For competition experiments, the competitive probes were added at a 100-fold molar excess. The EMSA was performed with the LightShift Chemiluminescent EMSA kit (Pierce).

\section{Results}

\section{Overexpression of $A t D R E B 1 A$ in soybean caused severe dwarfness and delayed flowering}

Transgenic lines of soybean cultivar Huachun 5 overexpressing AtDREBIA were developed using an Agrobacterium tumefaciens-mediated method described previously (Suo et al. 2012). The transgenic lines showed dwarfism 
Table 1 Information regarding selected flowering-related genes

\begin{tabular}{|c|c|c|c|c|}
\hline $\begin{array}{l}\text { Arabidopsis } \\
\text { locus ID }\end{array}$ & Abbreviation & Function & Pathway & Soybean homologous genes \\
\hline $\begin{array}{l}\text { AT1G65480/ } \\
\text { AT4G20370 }\end{array}$ & FT & $\begin{array}{l}\text { PEBP (phosphatidylethanolamine-binding protein) } \\
\text { family protein }\end{array}$ & Integrator & $\begin{array}{l}\text { Glyma02g07650,Glyma08g28470, } \\
\text { Glyma08g47810, Glyma08g47820 } \\
\text { Glyma16g04830,Glyma16g04840, } \\
\text { Glyma16g26660, Glyma16g26690 } \\
\text { Glyma19g28390, } \\
\text { Glyma19g28400,Glyma18g53670 }\end{array}$ \\
\hline AT3G57390 & AGL18 & AGAMOUS-like 18 & Integrator & Glyma02g33040 \\
\hline AT2G45660 & SOC1 & AGAMOUS-like 20 & Integrator & $\begin{array}{l}\text { Glyma03g02200,Glyma07g08830, } \\
\text { Glyma09g40230, Glyma18g45780 } \\
\text { Glyma05g03660 }\end{array}$ \\
\hline AT5G10140 & $\begin{array}{l}\text { FLC, } \\
\text { AGL25 }\end{array}$ & $\begin{array}{l}\text { K-box and MADS-box transcription factor family } \\
\text { protein }\end{array}$ & $\begin{array}{l}\text { Vernalization, } \\
\text { autonomous }\end{array}$ & Glyma05g28130 \\
\hline AT1G69120 & AP1 AGL7 & $\begin{array}{l}\text { K-box and MADS-box transcription factor family } \\
\text { protein }\end{array}$ & Photoperiod & $\begin{array}{l}\text { Glyma01g08150,Glyma02g13420, } \\
\text { Glyma08g36380, Glyma16g13070 }\end{array}$ \\
\hline AT4G36920 & AP2, FL1 & Integrase-type DNA-binding superfamily protein & Photoperiod & $\begin{array}{l}\text { Glyma01g39520,Glyma03g33470, } \\
\text { Glyma05g18170, Glyma10g22390 } \\
\text { Glyma11g05720, Glyma17g18640, } \\
\text { Glyma19g36200 }\end{array}$ \\
\hline AT2G28550 & RAP2.7 & related to AP2.7 & Photoperiod & $\begin{array}{l}\text { Glyma02g09600, Glyma11g15650, } \\
\text { Glyma12g07800, Glyma13g40470, } \\
\text { Glyma15g04930 }\end{array}$ \\
\hline AT5G62430 & CDF1 & cycling DOF factor 1 & Photoperiod & Glyma06g20950 \\
\hline AT4G08920 & CRY1 & cryptochrome 1 & Photoperiod & $\begin{array}{l}\text { Glyma04g11010, Glyma06g10830, } \\
\text { Glyma13g01810, Glyma14g35020 }\end{array}$ \\
\hline AT1G04400 & CRY2 & cryptochrome 2 & Photoperiod & $\begin{array}{l}\text { Glyma02g00830,Glyma10g32390, } \\
\text { Glyma18g07770, Glyma20g35220 }\end{array}$ \\
\hline AT5G03840 & TFL & PEBP family protein & Photoperiod & $\begin{array}{l}\text { Glyma03g35250,Glyma09g26550, } \\
\text { Glyma16g32080, Glyma19g37890 }\end{array}$ \\
\hline AT1G22770 & GI & gigantea protein $(\mathrm{GI})$ & Photoperiod & $\begin{array}{l}\text { Glyma09g07240, Glyma10g36600, } \\
\text { Glyma20g30980 }\end{array}$ \\
\hline AT4G16280 & FCA & RNA binding; abscisic acid binding & Autonomous & Glyma17g03960 \\
\hline AT3G10390 & FLD & $\begin{array}{l}\text { Flavin containing amine oxidoreductase family } \\
\text { protein }\end{array}$ & Autonomous & Glyma02g18610 \\
\hline AT2G43410 & FPA & RNA binding & Autonomous & $\begin{array}{l}\text { Glyma11g13490, Glyma12g05490, } \\
\text { Glyma13g42060, Glyma15g03330 }\end{array}$ \\
\hline AT2G19520 & FVE & $\begin{array}{l}\text { Transducin family protein/WD-40 repeat family } \\
\text { protein }\end{array}$ & Autonomous & $\begin{array}{l}\text { Glyma09g07120,Glyma13g42660, } \\
\text { Glyma15g02770, Glyma15g18450 }\end{array}$ \\
\hline AT5G13480 & FY & Transducin/WD40 repeat-like superfamily protein & Autonomous & Glyma13g26820, Glyma15g37830 \\
\hline AT4G02560 & LD & Homeodomain-like superfamily protein & Autonomous & Glyma03g36970, Glyma19g39620 \\
\hline AT3G18990 & VRN1 & AP2/B3-like transcriptional factor family protein & Vernalization & $\begin{array}{l}\text { Glyma01g11670, Glyma04g43620, } \\
\text { Glyma07g21160, Glyma08g44640 } \\
\text { Glyma08g44650, Glyma09g18790, } \\
\text { Glyma09g20280, Glyma11g13210 } \\
\text { Glyma11g13220, Glyma20g01130, } \\
\text { Glyma20g24220,Glyma12g05250 } \\
\text { Glyma16g05110, Glyma19g27950 }\end{array}$ \\
\hline AT3G24440 & VRN5 & Fibronectin type III domain-containing protein & Vernalization & $\begin{array}{l}\text { Glyma05g35280, Glyma07g09800, } \\
\text { Glyma08g04440, Glyma09g32010 }\end{array}$ \\
\hline AT5G57380 & VIN3 & Fibronectin type III domain-containing protein & Vernalization & Glyma17g07000 \\
\hline AT4G16845 & VRN2 & & Vernalization & Glyma11g03960,Glyma01g41460, \\
\hline
\end{tabular}


and delayed-flowering phenotypes. The dwarfism was recovered after treated with $144 \mu \mathrm{M}$ GA once a week for 3 consecutive weeks or $60 \mu \mathrm{M}$ GA three times in 1 week (Suo et al. 2012). However, the delayed-flowering phenotype could not be rescued (Online Resource 3). The delayed flowering of the transgenic plants was regardless of day length. Additionally, compared with WT soybean plants, the transgenic plants had fewer leaves under all day length conditions (Fig. 1). Under short day length conditions, WT plants flowered at 32 DAE with 13 leaves, while transgenic plants flowered at 46 DAE with 8 leaves. Under intermediate and long day length conditions, WT plants flowered at 60 DAE with 42 leaves and 53 DAE with 18 leaves, respectively (Table 2). However, the transgenic plants did not flower until 60 DAE under the intermediate and long day length conditions (Table 2).


Fig. 1 Phenotypes of AtDREB1A-overexpressing transgenic and WT soybean plants grown under different day length conditions. a Phenotypes of transgenic and WT soybean plants grown under $8 \mathrm{~h}$ light/ $16 \mathrm{~h}$ dark conditions at 35 days after emergence (DAE). b, c Magnified view of (a), flower buds formed in WT plants, but transgenic plants maintained their vegetative growth. d Phenotypes of transgenic

and WT soybean plants under $12 \mathrm{~h}$ light/12 $\mathrm{h}$ dark conditions at 60 DAE. e, f Magnified view of (d), WT plants flowered, but transgenic plants maintained their vegetative growth. $g$ Phenotypes of transgenic and WT soybean plants under $16 \mathrm{~h}$ light $/ 8 \mathrm{~h}$ dark conditions at 54 DAE. h, i Magnified view of (g), WT plants flowered, but transgenic plants maintained their vegetative growth 
Table 2 Number of leaves in transgenic and WT soybean plants grown under different day length conditions

\begin{tabular}{|c|c|c|c|c|c|c|}
\hline \multirow[t]{2}{*}{ DAE } & \multicolumn{2}{|l|}{$8 \mathrm{~h}$ light $/ 16 \mathrm{~h}$ dark } & \multicolumn{2}{|c|}{$12 \mathrm{~h}$ light/12 h dark } & \multicolumn{2}{|l|}{$16 \mathrm{~h}$ light $/ 8 \mathrm{~h}$ dark } \\
\hline & WT & $\mathrm{L} 2$ & WT & $\mathrm{L} 2$ & WT & L2 \\
\hline 11 & $1.34 \pm 0.33 \mathrm{a}$ & $1.00 \pm 0.00 \mathrm{a}$ & $1.67 \pm 0.33 \mathrm{a}$ & $1.00 \pm 0.00 \mathrm{a}$ & $1.34 \pm 0.33 \mathrm{a}$ & $1.00 \pm 0.00 \mathrm{a}$ \\
\hline 18 & $2.34 \pm 0.33 b$ & $2.00 \pm 0.00 \mathrm{~b}$ & $3.67 \pm 0.33 a$ & $2.67 \pm 0.33 b$ & $2.67 \pm 0.33 b$ & $2.00 \pm 0.00 \mathrm{~b}$ \\
\hline 25 & $4.33 \pm 0.33 b c$ & $3.33 \pm 0.33 d$ & $6.67 \pm 0.33 a$ & $4.67 \pm 0.33 b$ & $4.00 \pm 0.00 \mathrm{bc}$ & $3.33 \pm 0.33 \mathrm{~d}$ \\
\hline 32 & $7.00 \pm 0.58 b$ & $4.67 \pm 0.33 d$ & $12.30 \pm 0.33 a$ & $6.00 \pm 0.00 \mathrm{bc}$ & $7.00 \pm 0.58 b$ & $5.33 \pm 0.33 d$ \\
\hline 39 & $13.00 \pm 0.58 \mathrm{a}(\mathrm{F})$ & $6.67 \pm 0.33 c$ & $14.30 \pm 0.33 a$ & $7.67 \pm 0.33 b c$ & $13.00 \pm 0.58 \mathrm{a}$ & $9.00 \pm 0.58 b$ \\
\hline 46 & $13.00 \pm 0.58 c$ & $7.67 \pm 0.33 \mathrm{f}$ & $22.30 \pm 0.88 \mathrm{a}$ & $8.67 \pm 0.33 \mathrm{ef}$ & $15.00 \pm 0.00 b$ & $10.30 \pm 0.88 \mathrm{~d}$ \\
\hline 53 & $13.00 \pm 0.58 c$ & $8.00 \pm 0.00 \mathrm{~d}(\mathrm{~F})$ & $31.70 \pm 0.88 \mathrm{a}$ & $12.00 \pm 0.00 \mathrm{c}$ & $17.30 \pm 0.88 \mathrm{~b}(\mathrm{~F})$ & $13.00 \pm 0.58 \mathrm{c}$ \\
\hline 60 & $13.00 \pm 0.58 \mathrm{~d}$ & $8.00 \pm 0.00 \mathrm{e}$ & $42.30 \pm 0.88 \mathrm{a}(\mathrm{F})$ & $25.30 \pm 0.67 b$ & $17.30 \pm 0.88 c$ & $14.70 \pm 0.67 \mathrm{~d}$ \\
\hline
\end{tabular}

Values are the means of three biological replicates \pm standard error. Different letters in each row indicate significant differences as determined by the analysis of variance, $p<0.05$

$D A E$ days after emergence, $F$ flowering

\section{AtDREB1A overexpression affects the expression levels of flowering-related genes}

Overexpressing $D R E B$ genes often result in delayed flowering, but the molecular mechanism involved in this is little known (Huang et al.2009; Tong et al. 2009). In our study, the delayed-flowering phenomenon could not be recovered by the GA treatment, implying this flowering delay is GAindependent pathway. Thus, the transcriptional levels of major flowering-related genes of the other three flowering pathways and key flowering integrator genes were detected under different day length conditions in WT and transgenic plants, and 26 genes displayed differential expression patterns (Fig. 2).

In the vernalization pathway, the differentially expressed genes included the AtVRN1 homologs Glymallg13210, Glyma11g13220, Glyma20g01130, Glyma07g21160, and Glyma $12 g 05250$. We also analyzed the homologs of AtVRN2 (Glyma01 g41460), AtVRN3 (Glyma17g07000), and AtVRN5 (Glyma09g32010 and Glyma07g09800). Compared with WT plants, the transcriptional level of each of these genes was much higher in transgenic plants regardless of day length conditions. Especially, the transcriptional level of Glymallg13220 (designated as GmVRN1-like) was pronouncedly up-regulated, about 27-fold, 50-fold, 20-fold higher in transgenic soybean plants than that in WT plants under long, intermediate and short day condition, respectively. These results suggest a potential relationship between GmVRN1-like and AtDREBIA in terms of the delayedflowering phenotype (Fig. 2a).

In the autonomous flowering pathway, we identified the A. thaliana FVE homologs Glyma09g07120 and Glyma15g18450. We also examined the transcriptional levels of FCA homologs (Glyma12g05490, Glyma17g03960, and Glyma15g03330) and $F Y$ homologs (Glymal3g16820, Glyma15g37830, and Glyma19g39620). Compared with that in WT plants, these genes showed higher transcriptional levels in transgenic plants under all 3-day length conditions (Fig. 2b). The photoperiod floral meristem genes, including Glyma15g04930, Glyma13g40470, Glyma02g09600, and Glyma05g18170 of the AP2 family, were more highly expressed in transgenic soybean plants than in WT plants (Fig. 2c).

We also detected the transcriptional levels of soybean floral integrators, including a floral repressor $(F L C)$ and floral activators ( $F T$ and $S O C 1$ ). The $F L C$ had a higher, while the $F T$ and $S O C 1$ showed lower transcriptional levels in transgenic plants than those in WT plants (Fig. 2d).

\section{GmVRN1-like may be a direct downstream target of AtDREB1A in soybean}

The DREB transcription factors can specifically bind the DRE cis element with a core A/GCCGAC sequence presenting in the promoter of downstream genes (Stockinger et al. 1997; Gilmour et al. 1998; Liu et al.1998). The higher expression level of GmVRN1-like in transgenic plants than in WT plants, suggested that GmVRN1-like may be the downstream target of AtDREB1A. There is a motif with a core sequence of ACCGAC in the GmVRN1-like promoter region, 168 bp upstream of the start codon (Lü et al. 2015). This motif has $100 \%$ identity to the DRE cis-element present in the promoter of RD29A (Maruyama et al. 2004).

To confirm whether AtDREB1A can bind DRE motif in the GmVRN1-like promoter, EMSA experiments were conducted. Shifted bands were observed under the conditions of GST-AtDREB1A recombinant protein combined with $r d 29 A$ or GmVRN1-like probes. No shifted bands were observed under the conditions of probes only or probes combined with GST control protein (Fig. 3). Both of cold and mutated probes impaired the signal in a small degree. It turns out that AtDREB1A interacts with core sequence of 

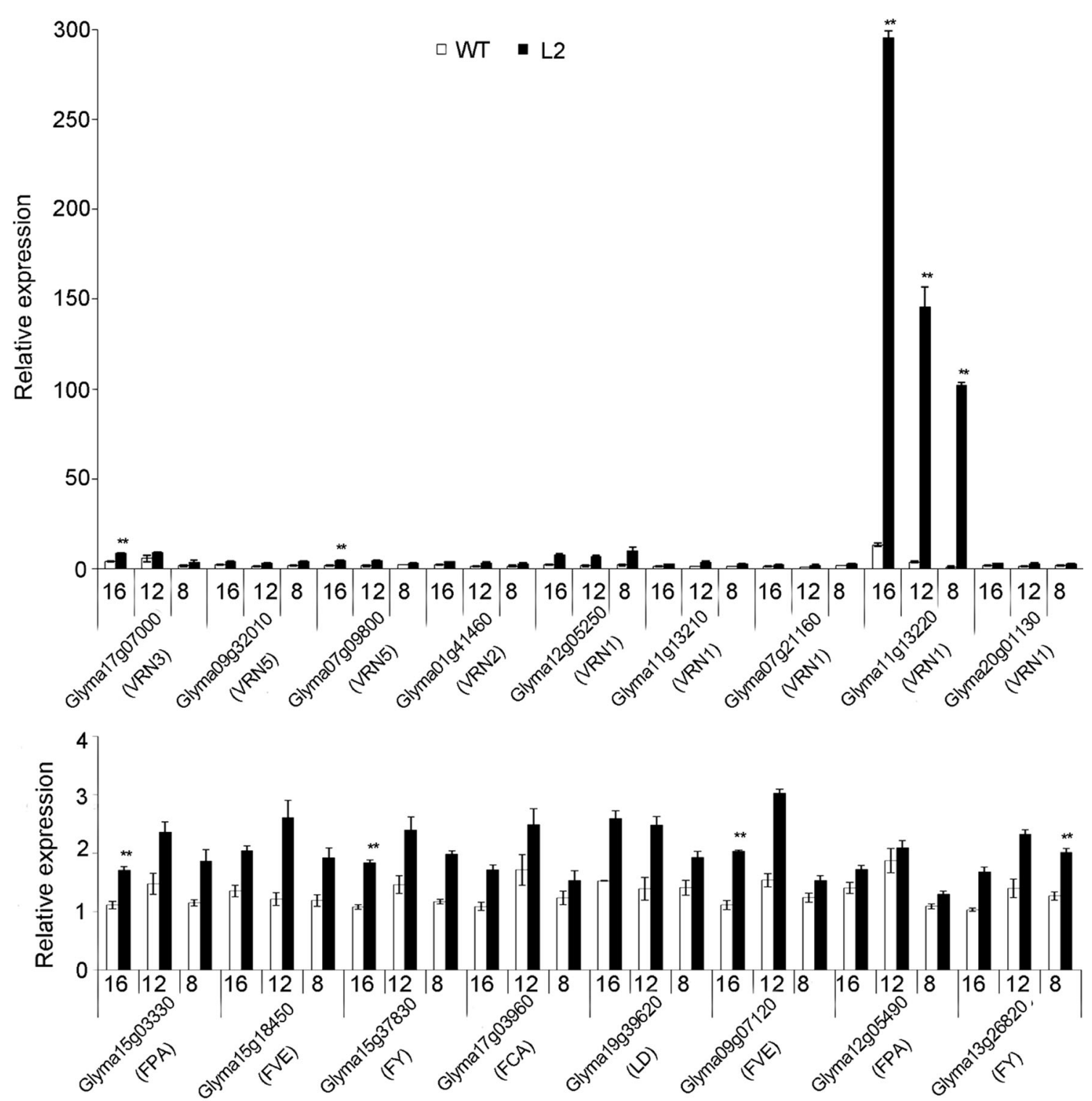

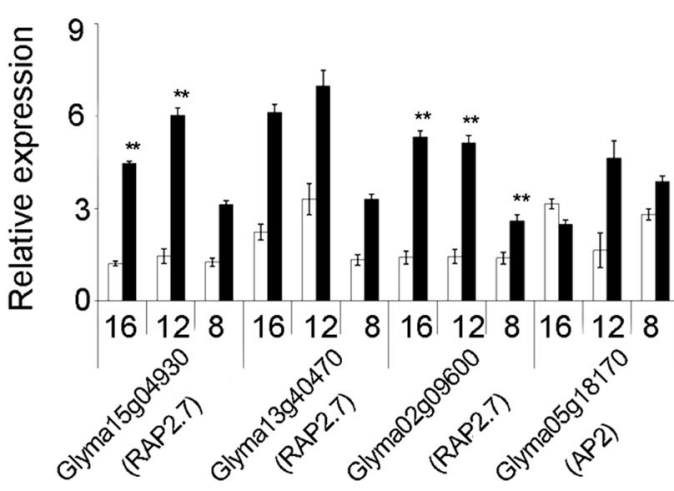

Fig. 2 Transcriptional levels of flowering-related genes a transcriptional levels of genes in the vernalization pathway. b Transcriptional levels of genes in the autonomous pathway. $\mathbf{c}$ Transcriptional levels of genes in the photoperiod pathway. d Transcriptional levels of floral

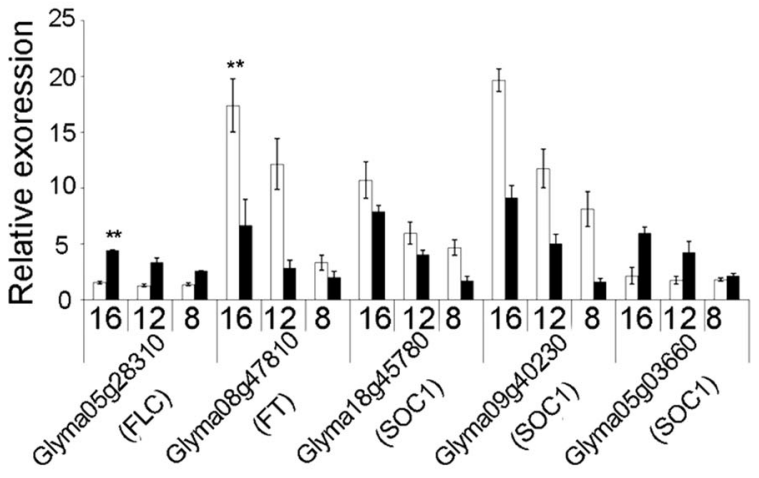

integrators. $\beta$-tubulin was used as an internal control. Values are the mean of three biological replicates \pm standard error. Student's $t$ test, $* * p<0.01$ 
DRE element present in the promoter of GmVRN1-like in vitro (Fig. 3).

\section{Discussion}

The delayed-flowering phenotypes caused by overexpression of AtDREB1B, GhDREB1, and AtDREBIF were rescued by GA treatments (Achard et al. 2008; Magome et al. 2008; Huang et al. 2009), suggesting that they are GAmetabolism dependent. However, delayed flowering cannot be reversed by exogenous GA treatment caused by overexpression of AtDREB1A or DgDREB1A (Tong et al. 2009; Suo et al. 2012), which means that other mechanisms may regulate the flowering of transgenic plants. In our previous study, overexpression of AtDREBlA gene in soybean resulting transgenic plants exhibited dwarfism with no observable internodes, darker green and had smaller leaves and seeds compared with the WT plants. The abnormal phenotypic characteristics could be reversed after exogenous GA treatments, we speculated that AtDREBIA mediates GA metabolism by regulating genes in GA synthesis and deactivation pathways (Suo et al. 2012).

Previous studies regarding delayed flowering focused only on the expression of key flowering regulators, including $F L C, F T$, and $C O$. For example, with respect to $D g D R E B 1 A$ - overexpressing A. thaliana plants, $C O$ and $F T$ were down-regulated, while the expression level of FLC was unaffected. This suggested that delayed flowering is associated with the photoperiod pathway (Sun et al. 2013). Moreover, FLC and $C O$ were affected in A. thaliana plants overexpressing $G h D R E B 1$, indicating that delayed flowering may be the result of changes to multiple flowering pathways (Huang et al. 2009). However, flowering is a complex process that is regulated by numerous genes. In soybean, 491 flowering regulatory genes belonging to the photoperiod, vernalization, autonomous, and GA flowering pathways have been identified by comparing the soybean and $A$. thaliana genomes (Jung et al. 2012). Thus, we analyzed the expression levels of major flowering genes from the photoperiod, vernalization, and autonomous pathways along with flowering integrators in AtDREB1Aoverexpressing transgenic and WT soybean plants. The expression level of GmVRN1-like, which is a homolog of the A. thaliana flowering vernalization gene (VRN1), was significantly $(P<0.01)$ higher in transgenic plants than that of WT plants (Fig. 2). Additionally, EMSA results revealed that $G m V R N 1$-like is a direct downstream target of AtDREB1A (Fig. 3). The FLC expression level was activated, while the $F T$ and $S O C 1$ expression levels were inhibited in transgenic plants (Fig. 2). This indicates that the late flowering of transgenic soybean may be linked to changes in the vernalization flowering pathway, despite soybean being a photoperiod-sensitive plant.

The overexpression of $D R E B$ genes causes delayed flowering because of the up-regulation of FLC expression. However, little is known about how $D R E B$ genes regulate $F L C$ expression (Seo et al. 2009). In this study, there was no evidence that AtDREBIA directly regulates $F L C$ expression, but the EMSA results indicated that AtDREB1A regulates GmVRN1-like expression by binding the cold response elements in the GmVRN1-like promoter region. Intermittent cold treatment is known to delay flowering through up-regulation of FLC expression (Kim et al. 2012). The overexpression of AtDREB 1A may simulate exposure to cold stress, thus activating the GmVRN1-like gene, which belongs to the vernalization flowering pathway.

In A. thaliana, FLC chromatin was inactivated initially by VIN3 production through histone modification (Sung and Amasino 2004). Thereafter, FLC chromatin structure
Fig. 3 EMSA results indicating AtDREB1A specifically bound to the DRE in vitro. Biotinlabeled DNA probes $(1 \mathrm{nmol})$ were combined with purified protein $(5 \mu \mathrm{g})$. The $r d 29 A$ labeled probe efficiently binds to the GST-AtDREB1A recombine protein. The GmVRN1-like labeled probe have shown the same results with that of $r d 29 A$ labeled probe, which indicated the AtDREB1A can bind the DRE cis-elemnt present in the promoter region

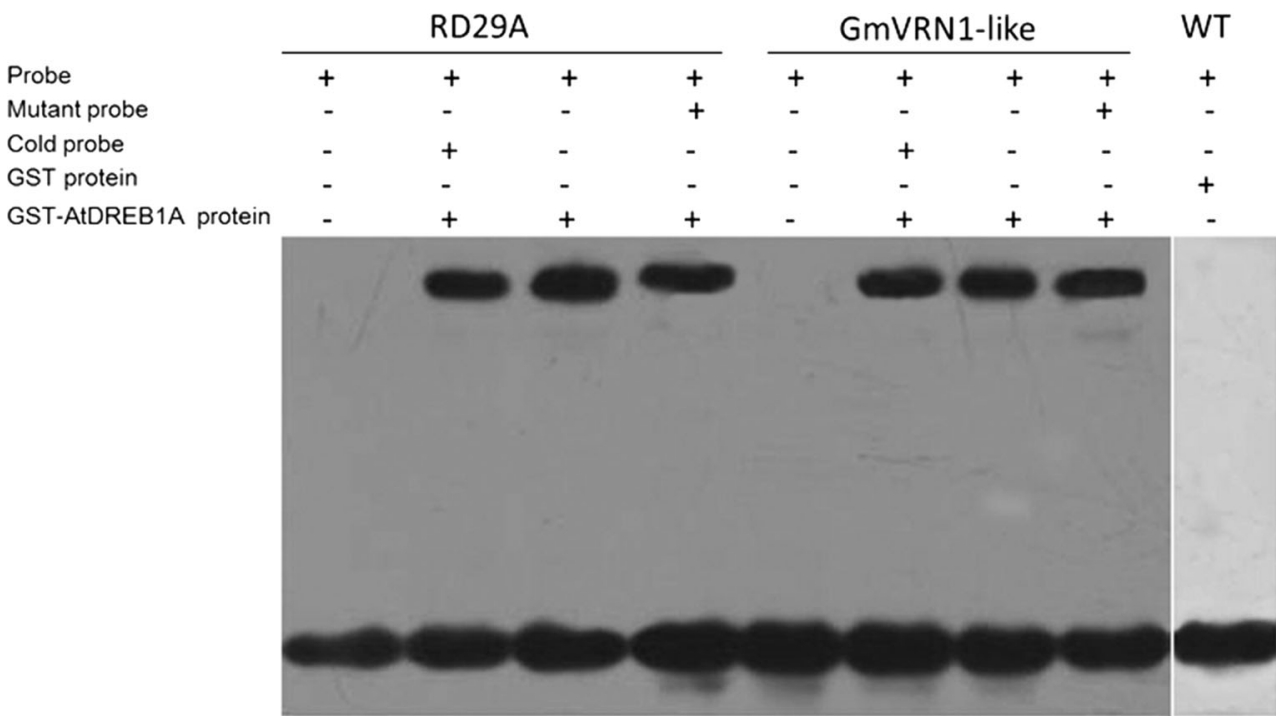


was permanently inactivated by VRN1, VRN2, and LHP1 via heterochromatin formation (Bastow et al. 2004; Sung and Amasino 2004; Seo et al. 2009). The FLC gene is regulated by many pathways, which are associated with different chromatin pathways and co-transcriptional mechanisms related to antisense transcripts called COOLAIR (cold-induced long antisense intragenic RNA) (Swiezewski et al. 2009; Sun et al. 2013). In soybean, we completed two-hybrid assays to preliminarily study the relationship between GmVRN1-like and GmFLC, but no interactions were observed (data not shown).

There has been limited research on soybean VRNI genes. In A. thaliana, VRN1 encodes a plant-specific protein (Levy et al. 2002). Overexpression of VRN1 causes early flowering in A. thaliana. However, vrnl mutants exhibit decreased vernalization response rather than delayed flowering (Levy et al. 2002). Low homology between GmVRN1-like and AtVRN1 suggests there are many differences in their functions (Lü et al. 2015). We have cloned GmVRN1-like and overexpressed it in A. thaliana and found it promotes flowering in transgenic plants (Lü et al. 2015). This further confirms the likely role of GmVRN1-like in the regulation of flowering.

Soybean is typically a photoperiod-sensitive plant. Comparative genomic analyses revealed that vernalization pathway genes are present in the soybean and A. thaliana genome (Schmutz et al. 2010; Jung et al. 2012). This indicates their potential role in soybean flowering. Our findings demonstrate that the overexpression of AtDREB1A activates GmVRN1-like expression in transgenic soybean plants. In A. thaliana, brief exposures to cold conditions before vernalization lead to CBF-activated FLC expression, which delays flowering and increases freezing tolerance (Seo et al. 2009). In wheat, $V R N-1$ negatively regulates the cold acclimation pathway (Chew and Halliday 2011). In our study, overexpression of AtDREB1A might mimic prolonged exposure to cold stress, which triggers the protective vernalization pathway to ensure flowering is arrested until more favorable conditions return. The vernalization pathway may be an alternative flowering pathway in soybean that is activated in specific situations, including exposure to stress.

\section{Conclusions}

Constitute overexpression of AtDREB1A result in soybean plants delayed flowering. The up-regulation of GmVRN1like expression may be responsible for this phenomenon. Our results suggest that although soybean is not a vernalization crop, the vernalization pathway may serve as an alternative flowering pathway that is activated in specific conditions.
Author contribution statement Conceived and designed the experiments: HCS, JL, HN. Performed the experiments: HCS, JL, QBM, CYY, XXZ, XM, SZH. Analyzed the data: HCS, JL, XM. Contributed reagents/materials/analysis tools: QBM, CYY, XXZ. Wrote the paper: HCS, JL, QBM, $\mathrm{HN}, \mathrm{SZH}$.

Acknowledgments Thanks to Dr. Qiaoying Zeng for comments and revisions to this article. This study was supported by the Major Projects of New Varieties Cultivation of Genetically Modified Organisms (2014ZX08004-002 and 2013ZX08004-002), the Special Funds for the Industrial Technology System of the National Modern Agriculture (CARS-04-PS09), the Research Project of The State Key Laboratory for Conservation and Utilization of Subtropical Agrobioresources, South China Agricultural University (4100-M13024), and the National Special Project for Transgenic Research (2014ZX0800921B).

Open Access This article is distributed under the terms of the Creative Commons Attribution 4.0 International License (http://crea tivecommons.org/licenses/by/4.0/), which permits unrestricted use, distribution, and reproduction in any medium, provided you give appropriate credit to the original author(s) and the source, provide a link to the Creative Commons license, and indicate if changes were made.

\section{References}

Achard P, Gong F, Cheminant S et al (2008) The cold-inducible $C B F 1$ factor-dependent signaling pathway modulates the accumulation of the growth-repressing DELLA proteins via its effect on gibberellin metabolism. Plant Cell 20:2117-2129

Agarwal PK, Agarwal P, Reddy MK et al (2006) Role of DREB transcription factors in abiotic and biotic stress tolerance in plants. Plant Cell Rep 25:1263-1274

Baker SS, Wilhelm KS, Thomashow MF (1994) The 5'-region of Arabidopsis thaliana cor15a has cis-acting elements that confer cold-, drought-and ABA-regulated gene expression. Plant Mol Biol 24:701-713

Bastow R, Mylne JS, Lister C et al (2004) Vernalization requires epigenetic silencing of FLC by histone methylation. Nature 427:164-167

Baurle I, Dean C (2006) The timing of developmental transitions in plants. Cell 125:655-664

Bernier G, Perilleux C (2005) A physiological overview of the genetics of flowering time control. Plant Biotechnol J 3:3-16

Blumel M, Dally N, Jung C (2015) Flowering time regulation in crops-what did we learn from Arabidopsis? Curr Opin Biotechnol 32:121-129

Boss PK, Bastow RM, Mylne JS et al (2004) Multiple pathways in the decision to flower: enabling, promoting, and resetting. Plant Cell 16(Suppl):S18-S31

Chen WJ, Zhu T (2004) Networks of transcription factors with roles in environmental stress response. Trends Plant Sci 9:591-596

Chew YH, Halliday KJ (2011) A stress-free walk from Arabidopsis to crops. Curr Opin Biotechnol 22:281-286

Dubouzet JG, Sakuma Y, Ito Y et al (2003) OsDREB genes in rice, Oryza sativa $L$., encode transcription activators that function in drought-, high-salt- and cold-responsive gene expression. Plant J 33:751-763

Gilmour SJ, Zarka DG, Stockinger EJ et al (1998) Low temperature regulation of the Arabidopsis CBF family of AP2 transcriptional 
activators as an early step in cold-induced COR gene expression. Plant J 16:433-442

Gilmour SJ, Sebolt AM, Salazar MP et al (2000) Overexpression of the Arabidopsis CBF3 transcriptional activator mimics multiple biochemical changes associated with cold acclimation. Plant Physiol 124:1854-1865

Gilmour SJ, Fowler SG, Thomashow MF (2004) Arabidopsis transcriptional activators $C B F 1, C B F 2$, and $C B F 3$ have matching functional activities. Plant Mol Biol 54:767-781

Hecht V, Foucher F, Ferrandiz C et al (2005) Conservation of Arabidopsis flowering genes in model legumes. Plant Physiol 137:1420-1434

Hsieh TH, Lee JT, Yang PT et al (2002) Heterology expression of the Arabidopsis C-repeat/dehydration response element binding factor 1 gene confers elevated tolerance to chilling and oxidative stresses in transgenic tomato. Plant Physiol 129:1086-1094

Huang JG, Yang M, Liu P et al (2009) GhDREB1 enhances abiotic stress tolerance, delays GA-mediated development and represses cytokinin signalling in transgenic Arabidopsis. Plant Cell Environ 32:1132-1145

Ito Y, Katsura K, Maruyama K et al (2006) Functional analysis of rice $D R E B 1 / C B F$-type transcription factors involved in cold-responsive gene expression in transgenic rice. Plant Cell Physiol 47:141-153

Jung CH, Wong CE, Singh MB et al (2012) Comparative genomic analysis of soybean flowering genes. PLoS One 7:e38250

Kasuga M, Miura S, Shinozaki K et al (2004) A combination of the Arabidopsis DREB1A gene and stress-inducible $r d 29 A$ promoter improved drought- and low-temperature stress tolerance in tobacco by gene transfer. Plant Cell Physiol 45:346-350

Kim MY, Shin JH, Kang YJ et al (2012) Divergence of flowering genes in soybean. J Biosci 37:857-870

Kissoudis C, van de Wiel C, Visser R et al (2014) Enhancing crop resilience to combined abiotic and biotic stress through the dissection of physiological and molecular crosstalk. Front Plant Sci 5:207

Lata C, Prasad M (2011) Role of DREBs in regulation of abiotic stress responses in plants. J Exp Bot 62:4731-4748

Lee H, Suh SS, Park E et al (2000) The AGAMOUS-LIKE 20 MADS domain protein integrates floral inductive pathways in Arabidopsis. Genes Dev 14:2366-2376

Levy YY, Mesnage S, Mylne JS et al (2002) Multiple roles of Arabidopsis VRN1 in vernalization and flowering time control. Science 297:243-246

Li J, Wei SM, Ouyang B et al (2012) Tomato SIDREB gene restricts leaf expansion and internode elongation by downregulating key genes for gibberellin biosynthesis. J Exp Bot 63:6407-6420

Liu Q, Kasuga M, Sakuma Y et al (1998) Two transcription factors, DREB1 and DREB2, with an EREBP/AP2 DNA binding domain separate two cellular signal transduction pathways in droughtand low-temperature-responsive gene expression, respectively, in Arabidopsis. Plant Cell 10:1391-1406

Livak KJ, Schmittgen TD (2001) Analysis of relative gene expression data using real-time quantitative PCR and the 2(-Delta Delta $\mathrm{C}(\mathrm{T})$ ) method. Methods 25:402-408

Lü J, Suo H, Yi R et al (2015) Glyma11g13220, a homolog of the vernalization pathway gene VERNALIZATION 1 from soybean [Glycine max (L.) Merr.], promotes flowering in Arabidopsis thaliana. BMC Plant Biol. doi:10.1186/s12870-015-0602-6

Magome H, Yamaguchi S, Hanada A et al (2004) dwarf and delayedflowering 1, a novel Arabidopsis mutant deficient in gibberellin biosynthesis because of overexpression of a putative AP2 transcription factor. Plant J 37:720-729

Magome H, Yamaguchi S, Hanada A et al (2008) The DDF1 transcriptional activator upregulates expression of a gibberellindeactivating gene, GA2ox7, under high-salinity stress in Arabidopsis. Plant J 56:613-626
Maruyama K, Sakuma Y, Kasuga M et al (2004) Identification of cold-inducible downstream genes of the Arabidopsis DREB1A/ CBF3 transcriptional factor using two microarray systems. Plant J 38:982-993

Michaels SD, Amasino RM (1999) FLOWERING LOCUS C encodes a novel MADS domain protein that acts as a repressor of flowering. Plant Cell 11:949-956

Mizoi J, Shinozaki K, Yamaguchi-Shinozaki K (2012) AP2/ERF family transcription factors in plant abiotic stress responses. Biochim Biophys Acta 1819:86-96

Moon J, Lee H, Kim M et al (2005) Analysis of flowering pathway integrators in Arabidopsis. Plant Cell Physiol 46:292-299

Mouradov A, Cremer F, Coupland G (2002) Control of flowering time: interacting pathways as a basis for diversity. Plant Cell 14(Suppl):S111-S130

Sakuma Y, Liu Q, Dubouzet JG et al (2002) DNA-binding specificity of the ERF/AP2 domain of Arabidopsis DREBs, transcription factors involved in dehydration- and cold-inducible gene expression. Biochem Biophys Res Commun 290:998-1009

Schmutz J, Cannon SB, Schlueter J et al (2010) Genome sequence of the palaeopolyploid soybean. Nature 463:178-183

Seo E, Lee H, Jeon J et al (2009) Crosstalk between cold response and flowering in Arabidopsis is mediated through the flowering-time gene $S O C 1$ and its upstream negative regulator $F L C$. Plant Cell 21:3185-3197

Simpson GG, Dean C (2002) Arabidopsis, the Rosetta stone of flowering time? Science 296:285-289

Srikanth A, Schmid M (2011) Regulation of flowering time: all roads lead to Rome. Cell Mol Life Sci 68:2013-2037

Stockinger EJ, Gilmour SJ, Thomashow MF (1997) Arabidopsis thaliana $\mathrm{CBF} 1$ encodes an AP2 domain-containing transcriptional activator that binds to the C-repeat/DRE, a cis-acting DNA regulatory element that stimulates transcription in response to low temperature and water deficit. Proc Natl Acad Sci USA 94:1035-1040

Sun Q, Csorba T, Skourti-Stathaki K et al (2013) R-loop stabilization represses antisense transcription at the Arabidopsis FLC locus. Science 340:619-621

Sung S, Amasino RM (2004) Vernalization and epigenetics: how plants remember winter. Curr Opin Plant Biol 7:4-10

Suo H, Ma Q, Ye K et al (2012) Overexpression of AtDREB1A causes a severe dwarf phenotype by decreasing endogenous gibberellin levels in soybean [Glycine max (L.) Merr]. PLoS One 7:e45568

Swiezewski S, Liu F, Magusin A et al (2009) Cold-induced silencing by long antisense transcripts of an Arabidopsis Polycomb target. Nature 462:799-802

Thomashow MF (1998) Role of cold-responsive genes in plant freezing tolerance. Plant Physiol 118:1-8

Thomashow MF (1999) Plant cold acclimation: freezing tolernce genes and regulatory mechanisms. Annu Rev Plant Physiol Plant Mol Biol 50:571-599

Tong Z, Hong B, Yang Y et al (2009) Overexpression of two chrysanthemum $D g D R E B 1$ group genes causing delayed flowering or dwarfism in Arabidopsis. Plant Mol Biol 71:115-129

Wang Y, Yu K, Poysa V et al (2012) Selection of reference genes for normalization of qRT-PCR analysis of differentially expressed genes in soybean exposed to cadmium. Mol Biol Rep 39:1585-1594

Watanabe S, Harada K, Abe J (2012) Genetic and molecular bases of photoperiod responses of flowering in soybean. Breed Sci 61:531-543

Yamaguchi-Shinozaki K, Shinozaki K (1994) A novel cis-acting element in an Arabidopsis gene is involved in responsiveness to drought, low-temperature, or high-salt stress. Plant Cell 6:251-264

Yamaguchi-Shinozaki K, Shinozaki K (2006) Transcriptional regulatory networks in cellular responses and tolerance to dehydration and cold stresses. Annu Rev Plant Biol 57:781-803 